\title{
Astronomy for a Better World: IAU Office of Astronomy for Development Activities to Grow and Advance Astronomy Education and Research at Universities in the Developing World
}

\author{
Edward F. Guinan ${ }^{1}$ and Katrien Kolenberg ${ }^{2,3,4}$ \\ ${ }^{1}$ Astronomy \& Astrophysics Department, Villanova University, \\ 800 Lancaster Ave, Villanova PA 19085, USA, email: edward.guinan@villanova.edu \\ ${ }^{2}$ Physics Department, University of Antwerp, Groenenborgerlaan 171, 2020 Antwerp, Belgium \\ ${ }^{3}$ Harvard-Smithsonian Center for Astrophysics, 60 Garden Street, Cambridge MA 02138, USA \\ email: kkolenberg@cfa.harvard.edu \\ ${ }^{4}$ Institute of Astronomy, KU Leuven, Celestijnenlaan 200D, 3001 Heverlee, Belgium
}

\begin{abstract}
In 2012, the International Astronomical Union (IAU), through its Office of Astronomy for Development (OAD), established the three Task Forces which drive global activities using astronomy as a tool to stimulate development. These Task Forces are: (i) Astronomy for Universities and Research; (ii) Astronomy for Children and Schools; and (iii) Astronomy for the Public.
\end{abstract}

Keywords. astronomy, development, strategic plan

\section{Astronomy for Development at the Universities \& Research level}

Task Force One (TF-1) on Astronomy for Universities \& Research was established in 2012 as part of the IAU Office of Astronomy for Development (OAD). This Task Force drives activities related to astronomy education and research at universities mainly focused on the developing world. Astronomy is used to stimulate research and education in STEM fields and to develop and promote astronomy in regions of the world where there is little or no astronomy. There is also potential for developing research in the historical and cultural aspects of astronomy which may prove important for stimulating an interest in the subject in communities where there is yet no established interest in the science. Since the establishment of the OAD, twenty-six TF-1 programs have been funded to support a wide variety of interesting and innovative astronomy programs in Africa, Asia, South-East Asia, the Middle-East, and in South \& Central America. Nearly every aspect of development has been supported. These programs include supporting: regional astronomy training schools, specialized workshops, research visits, exchange visits, university twinning programs, distance learning projects, university astronomy curriculum development, as well as small telescope and equipment grants.

Also supported within the TF-1 program framework are Visiting Astronomer \& Exchange programs; Sabbatical Visits; funds for travel of astronomer to developing countryairfare / small stipend; Development of distance learning packages for University Astronomy Courses; Regional Specialized /Focused Workshops \& Schools, Start-up funds for Association of ISYA Alumni Technology Internships e.g. instrument specialists to consult; Telescope adoption programs in which existing observatories "adopt" a facility in a 
developing region; Collaborations with institutions where a percentage of research grants are spent on development; Open source text book for astronomy education at all levels and many others that related to the development of Astronomy education and research at the university level.

As examples, the TF-1 programs approved for 2015 (Ten programs approved for support/ partial support) are given below. Because of the limited amount of funds, there are many valuable programs that could not be funded. Fund raising is being carried out to support these other highly ranked programs. The over subscription rate for TF-1 proposals over the last three years is about $4 / 1$.

2015 (Ten programs approved for support/ partial support)

- Visiting Program at the Harvard-Smithsonian Center for Astrophysics (USA-SA)

- Time Variability in Modern Astrophysics (Thailand)

- Latin American School of Observational Astronomy

- Summer School on Statistical Data Analysis and Data Mining in Astronomy

- Optical camera for a 14-inch telescope in Namibia (Namibia)

- Astronomy for Africa: Student Support - Astronomy Modules via Distance Learning

- National School on Astrophysical Simulation

- Introducing Data Analysis in the University System

- TARA, Fergusson College Node, Pune, India

- Guatemalan School of Astrophysics 2015

- Andean Cosmology School

In addition, a new multi-year program - Starlab - was introduced by TF1-members, J-P De Greve and Michele Gerbaldi, and proven successful through initial funds by OAD in 2013 and 2014 to bring "starlight" into the class room. In this program, students carry out and reduce CCD photometry secured by them using remotely controlled telescopes around the world. The primary goal is to develop the Astrolab program into a standard tool for astronomy education, useful for training in institutions and locations without easy telescope access. Pilot programs have already been carried out in Uganda, Rwanda and Ethiopia.

The following proposal selections criteria are considered in the selection of new projects: Alignment with the goals of IAU's 10 year strategic plan; Quality and relevance of content. Innovativeness and creativity of approach; Clear timeline indicators; Cost effectiveness of the project including a detailed budget; Potential to raise funding from other sources; Range of stakeholder participation (e.g. partnerships universities / industry / government); Degree of impact of the project with potential for sustainability.

Information and advice are provided about applying for support in the future. Additional information is available on the OAD website at www.astro4dev.org/. The next OAD call for proposals will be during mid-2016 for programs to be carried out in 2017 . Formal submission of proposals is typically due during September and the proposal evaluation outcomes are released during December. The OAD is continuously improving and expanding these programs to bring astronomy education and research to a greater number of people and indeed to use Astronomy for a Better World.

\section{References}

IAU 2012, http://www.iau.org/static/education/strategicplan_2010-2020.pdf 